\title{
Nuclear androgen receptor staining in bone metastases is related to a poor outcome in prostate cancer patients
}

\author{
S Crnalic ${ }^{1}, E_{1}$ Hörnberg $^{2}, P_{\text {Wikström }}^{2}, \bigcup_{2} H$ Lerner $^{3}$, A Tieva $^{4}$, \\ $O$ Svensson $^{1}, A$ Widmark ${ }^{4}$ and $A$ Bergh ${ }^{2}$
}

Departments of ${ }^{1}$ Orthopedics, ${ }^{2}$ Pathology, ${ }^{3}$ Odontology and ${ }^{4}$ Oncology, Umeå University, 90187 Umeå, Sweden

(Correspondence should be addressed to A Bergh; Email: anders.bergh@medbio.umu.se)

\begin{abstract}
Androgen receptors (ARs) are probably of importance during all phases of prostate cancer (PC) growth, but their role in bone metastases is largely unexplored. Bone metastases were therefore collected from hormone-naive $(n=11)$, short-term castrated $(n=7)$ and castration-resistant PC (CRPC, $n=44)$ patients by biopsy $(n=4)$ or at surgery to alleviate symptoms from metastases complications (metastasis surgery, $n=58$ ), and immunostained for nuclear ARs, Ki67, active caspase-3, prostate-specific antigen (PSA) and chromogranin A, and results were related to serum PSA, treatments and outcome. Nuclear AR immunostaining was decreased and apoptosis was increased, but cell proliferation remained largely unaffected in metastases within a few days after surgical castration. In CRPC patients, nuclear AR staining of metastases was increased when compared to short-term castrated patients. The nuclear AR staining score was related to tumour cell proliferation, but it was not associated with other downstream effects of AR activation such as apoptosis and PSA staining, and it was only marginally related to the presence of neuroendocrine tumour cells. Serum PSA at metastasis surgery, although related to outcome, was not associated with AR staining, markers of metastasis growth or PSA staining in metastases. High nuclear AR immunostaining was associated with a particularly poor prognosis after metastasis surgery in CRPC patients, suggesting that such men may benefit from the potent AR blockers now tested in clinical trials.
\end{abstract}

Endocrine-Related Cancer (2010) 17 885-895

\section{Introduction}

Metastasis to the bone is one of the most clinically important features of prostate cancer (PC). The standard therapy for bone metastases is androgen ablation where circulating androgens are reduced by surgical or medical castration. This therapy initially reduces symptoms and metastases growth, but after some time, the metastases relapse to castration-resistant growth (Denmeade \& Isaacs 2002). The mechanisms regulating growth of $\mathrm{PC}$ bone metastases as well as the mechanisms behind the early treatment response and the later relapse in androgen ablated patients are not fully established (Chen et al. 2004, Morrissey \& Vessella 2007, Ohlson et al. 2007, Wikstrom et al. 2009, Bonkhoff \& Berges 2010). Notably, most of our current knowledge about PC is based on studies of primary tumours or soft tissue metastases, and not on studies of bone metastases. The reason to this is that bone metastases are generally not resected and bone biopsies are seldom taken, except by a limited number of investigators who have performed autopsies to acquire samples and others who obtained tissue at surgery for stabilization of pathological fractures (Hobisch et al. 1995, Asmann et al. 2002, Cheville et al. 2002, Roudier et al. 2003, Shah et al. 2004).

Tumours relapsing after castration therapy were previously called androgen-independent, but recent studies show that the androgen receptor (AR) is often active also in what is now mostly referred to as castration-resistant PC (CRPC). Active AR signalling in CRPC could be caused by increased local synthesis of androgens, genetic or epigenetic changes in the ARs 
making it more sensitive to activation by androgens and other ligands, or expression of constitutively active AR variants, but the mechanisms involved are not fully understood (Chen et al. 2004, Holzbeierlein et al. 2004, Stanbrough et al. 2006, Montgomery et al. 2008, Guo et al. 2009, Hu et al. 2009). If bone metastases growth in CRPC patients is driven by AR signalling, those patients should probably benefit from treatment with new drugs that effectively inhibit the ARs (Tran et al. 2009). However, PC stem cells and neuroendocrine (NE) cells lack ARs, and if they constitute a substantial portion of the bone metastases, such patients should probably be treated by other means (Maitland \& Collins 2008).

To explore AR expression and possible downstream effects of the ARs such as cell proliferation, apoptosis and prostate-specific antigen (PSA) expression in PC bone metastases, we collected tissue at surgery for metastasis complications and examined it by immunohistochemisty. Most of the men had previously been treated with androgen ablation and occasionally also with radiation and chemotherapy, whereas others were previously untreated. We found that high AR staining was related to a particularly poor outcome after metastasis surgery in CRPC patients, but additional studies in larger materials are needed to confirm this conclusion.

\section{Materials and methods}

\section{Patients}

Fifty-eight men with metastatic PC who had surgery for metastatic spinal cord compression $(n=54)$ or pathological fractures of the femur $(n=4)$ between 2003 and 2009, from here on termed metastasis surgery, were included in the study. Patient characteristics and treatments are summarized in Table 1. From 16 patients, we retrieved biopsies from the primary tumours. In addition, we had access to paired computer tomography (CT)-guided vertebral needle biopsies from two patients taken immediately before and 3 days after surgical castration. The patients gave informed consent, and the study was approved by local ethical review board.

\section{Immunohistochemistry}

Samples were frozen in liquid nitrogen or fixed in buffered formalin, decalcified in $20 \%$ formic acid at $37{ }^{\circ} \mathrm{C}$ for $1-3$ days depending on the size of the specimen and embedded in paraffin. Paraffin sections $(5 \mu \mathrm{m})$ were stained with haematoxylin-eosin, and immunostained for ARs (PG-21, Upstate, Lake Placid, NY, USA), PSA (A0562, DAKO, Stockholm, Sweden), activated caspase-3 (Cell Signalling, Danvers, MA, USA) and Ki67 (MIB1, DAKO) as

Table 1 Clinical characteristics of prostate cancer bone metastases obtained at orthopaedic surgery. Continuous values are given as median (min-max values)

\begin{tabular}{|c|c|c|c|}
\hline Clinical characteristics & $\begin{array}{l}\text { Hormone- } \\
\text { naive } n=9\end{array}$ & $\begin{array}{l}\text { Short-term- } \\
\text { treated }^{\mathrm{a}} n=5\end{array}$ & $\begin{array}{l}\text { Castration- } \\
\text { resistant }{ }^{\mathrm{b}} n=44\end{array}$ \\
\hline Age at PCa diagnosis (years) & $80(60-85)$ & $76(72-88)$ & $69(51-81)$ \\
\hline Age at surgery (years) & - & - & $72(54-88)$ \\
\hline Serum PSA at PCa diagnosis (ng/ml) & $114(21-2550)$ & $935(124-4000)$ & $74(2-7300)$ \\
\hline Serum PSA at surgery $(\mathrm{ng} / \mathrm{ml})$ & - & - & $190(0-5140)$ \\
\hline \multicolumn{4}{|l|}{ Androgen deprivation therapy prior to surgery } \\
\hline Castration $^{\mathrm{c}}$ & - & 5 & 32 \\
\hline Castration plus anti-androgen $^{d}$ & - & 0 & 12 \\
\hline \multicolumn{4}{|l|}{ Radiation prior to surgery ${ }^{\mathrm{e}}$} \\
\hline Yes & 0 & 0 & 7 \\
\hline No & 9 & 5 & 37 \\
\hline \multicolumn{4}{|l|}{ Chemotherapy prior to surgery } \\
\hline Yes & 0 & 0 & 6 \\
\hline No & 9 & 5 & 38 \\
\hline Follow-up after surgery ${ }^{f}$ (months) & $29(7-69)$ & $13(0-41)$ & $6.0(0.25-56)$ \\
\hline Primary tumours available & 2 & 0 & 14 \\
\hline Time between primary tumour biopsy and surgery (years) & 0 & - & $4.5(1.4-12)$ \\
\hline
\end{tabular}

ashort-term-treated patients had received androgen ablation therapy for 2-7 days before orthopaedic surgery.

${ }^{\mathrm{b}}$ Castration-resistant patients had disease progression after long-term androgen deprivation therapy.

${ }^{c}$ Includes surgical ablation, LHRH/GnRH agonist therapy and therapy with anti-androgens (bicalutamide and flutamide), but specifically no anti-androgens at the time for orthopaedic surgery.

${ }^{\mathrm{d}}$ Castration therapy as stated above and anti-androgen therapy (bicalutamide) at the time for orthopaedic surgery.

${ }^{\text {e}}$ Radiation towards operation site.

fThe time between date of operation and the latest follow-up examination or death. 
described earlier (Ohlson et al. 2005, 2006, 2007). Sections were also immunostained for chromogranin A (5H7, 1:500, Novacastra, Leica Microsystems, Kista, Sweden). To validate the AR staining of decalcified paraffin-embedded sections, corresponding cryostat sections were stained with the same Upstate AR antibody, and in addition, cryostat and paraffin sections were stained with another AR antibody (265M, 1:200 Biogenex, San Ramon, CA, USA). The cryostat sections were fixed in $4 \%$ formaldehyde solution for $10 \mathrm{~min}$ and immunostained following the protocol as above, except that antigen retrieval was not used. Both AR antibodies used are directed against the $\mathrm{N}$-terminal non-ligand binding part of the ARs. Similarly, the Ki67 and PSA immunostainings were compared in paired paraffin and cryostat sections. In addition, all antibodies used were tested in multiple paraffinembedded samples from one patient with benign prostatic hyperplasia where the samples had been decalcified in formic acid as above for $0,1,3$ or 7 days. As negative controls, we excluded the primary antibodies from the staining protocols. Proliferation cells in the bone marrow served as a positive control for Ki67 and non-malignant prostate glands for PSA and chromogranin A, and 3-day castrated human prostate tumour tissue (Ohlson et al. 2005) for caspase-3.

The percentage of apoptotic (caspase-3-positive cells or cells showing the nuclear morphology of apoptosis in hematoxylin-eosin-stained sections) and proliferating (Ki67-positive) tumour epithelial cells was scored by evaluating 300-1000 cells per patient, as described earlier (Ohlson et al. 2005, 2007). The PSA staining and AR (Upstate antibody) staining were quantified by scoring the intensity ( 0 , no staining; 1 , weak; 2 , moderate; and 3 , intense staining) and the percentage of tumour cells stained $(1,1-25 \%$; 2 , 26-50\%; 3, 51-75\%; and 4, 76-100\%). A combined staining score, ranging from 0 to 12 , was then calculated by multiplying intensity with distribution. Occasional cases were excluded from analysis because of lack of staining in the positive control (for Ki67), too few tumour cells to count (for caspase-3) and a missing paraffin block (for Ki67, caspase-3 and ARs).

\section{Statistical analysis}

Correlations between variables were analysed using Spearman rank test. Groups were compared with the Mann-Whitney $U$ test. Paired observations were compared using the Wilcoxon test. Kaplan-Meier survival analysis was performed with death of PC as event and follow-up time as time between operation and the latest follow-up examination. Groups were compared with the log rank test. Multiple Cox survival analysis was performed for evaluation of independent predictive variables. A $P$ value $\leq 0.05$ was considered statistically significant. Statistical analysis was performed using the SPSS 17.0 software (SPSS Inc., Chicago, IL, USA).

\section{Results}

\section{Bone metastasis morphology}

In most metastases, no haematopoietic bone marrow remained. The marrow spaces were instead filled with sheets of tumour epithelial cells and a more or less prominent connective tissue stroma. Only occasionally, the tumour cells formed glandular acini, and almost all metastases were therefore scored as Gleason score $8-10$. The adjacent bone tissue shows mainly areas with either lamellar old bone or woven new bone with minor signs of bone resorption.
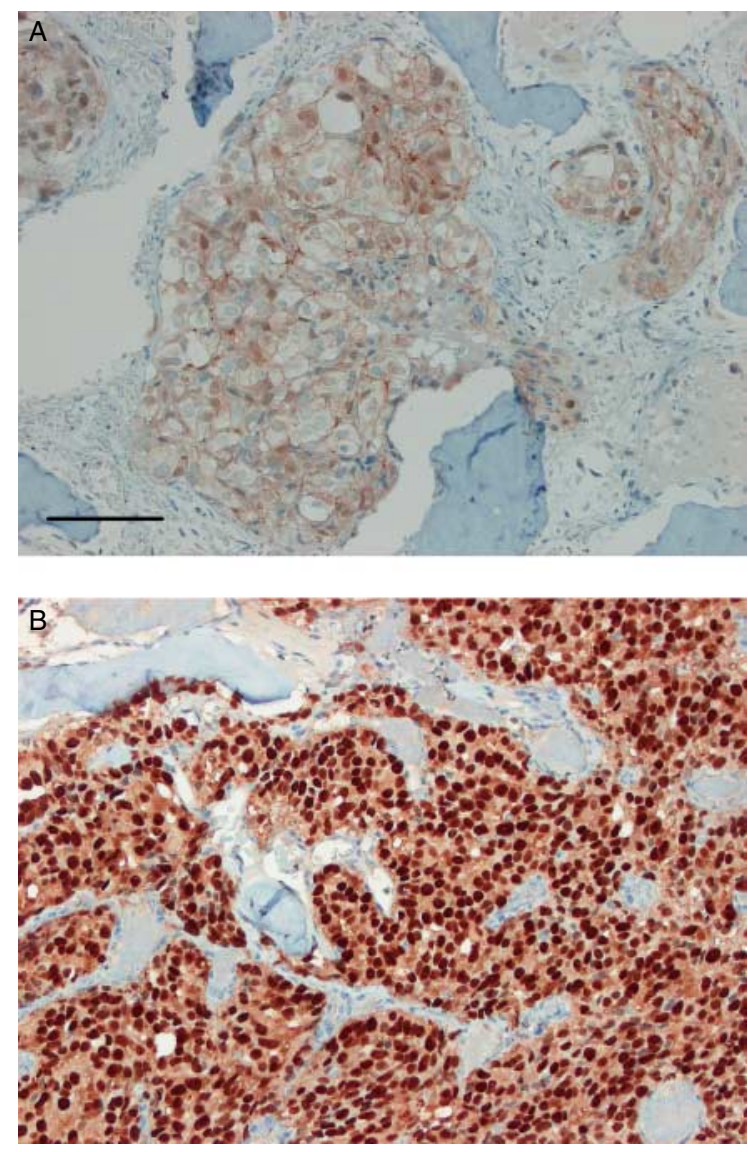

Figure 1 Prostate cancer bone metastases immunostained for the androgen receptor (AR). In some tumours, only few cells show weak AR staining (A), whereas in others, most cells show intense nuclear staining (B). Bar indicates $100 \mu \mathrm{m}$. 


\section{AR immunostaining in bone metastases}

As most of the available metastasis tissue was decalcified and paraffin embedded, we first tested whether this affected immunostaining intensity or distribution (see Supplementary information, see section on supplementary data given at the end of this article). In summary, our experiments showed that the AR antibody from Upstate, and the Ki67, PSA, chromogranin A and caspase-3 antibodies worked satisfactory in tissue decalcified up to 3 days (Supplementary Figures S1 and S2, see section on supplementary data given at the end of this article).

Most tumour epithelial cells in the bone metastases showed nuclear AR immunostaining (median distribution score was 4), but generally it was also possible to find tumour cells that lacked AR staining (Figs 1-3). Total lack of AR staining was only observed in two cases. Low AR staining in such cases was also found when cryostat sections were stained from these individuals. In cases with positive AR staining, the intensity ranged from weak to intense (median intensity score was 2, Fig. 3). No difference in tumour epithelial AR staining scores was observed between hormone-naive and CRPC metastases (Table 2). Treatment with bicalutamide or flutamide could possibly induce AR translocation to the nucleus, binding to AR-responsive elements, but AR staining in metastases treated with androgen ablation plus antiandrogens did not differ from metastases treated with androgen ablation only (Table 3). Chemotherapy or radiation towards operation site did also not influence the nuclear AR staining score (Table 3). The tumour metastasis stroma, including that in hormone-naive cases, generally lacked AR staining.

\section{Nuclear AR immunostaining of tumour epithelial cells in CRPC bone metastases is related to poor outcome}

First, we tested the robustness of the immunohistochemisty scoring. Two investigators (S C and A B) scored metastases independently. The score values obtained were highly correlated, and we therefore used the score values from the first author in survival analysis (see Supplementary information). Having noticed that the AR staining differed with respect to both distribution and intensity among patients with CRPC ( $n=44$, Fig. 3), it was of interest to see whether this and the combined AR staining scores were related to outcome after metastasis surgery. In total, 28 (63\%) of the CRPC patients showed high nuclear AR staining scores (a combined score of median or above; 8-12, from here on referred to as 'AR-high'), and they had significantly shorter cancer-specific survival than the AR-low patients (Fig. 4). Similarly, the 18 patients with maximal AR score did worse than the rest $(P=0.025$, data not shown). Most of the prognostic information derived from the combined AR staining score was related to the distribution score. A distribution above median ( $>75 \%$ of tumour cells stained) was related to a poor outcome as compared to the group of patients with a distribution score below median ( $P=0.006$, data not shown). As the number of hormone-naive patients was low, we did not examine the prognostic importance of AR staining for this group of patients.
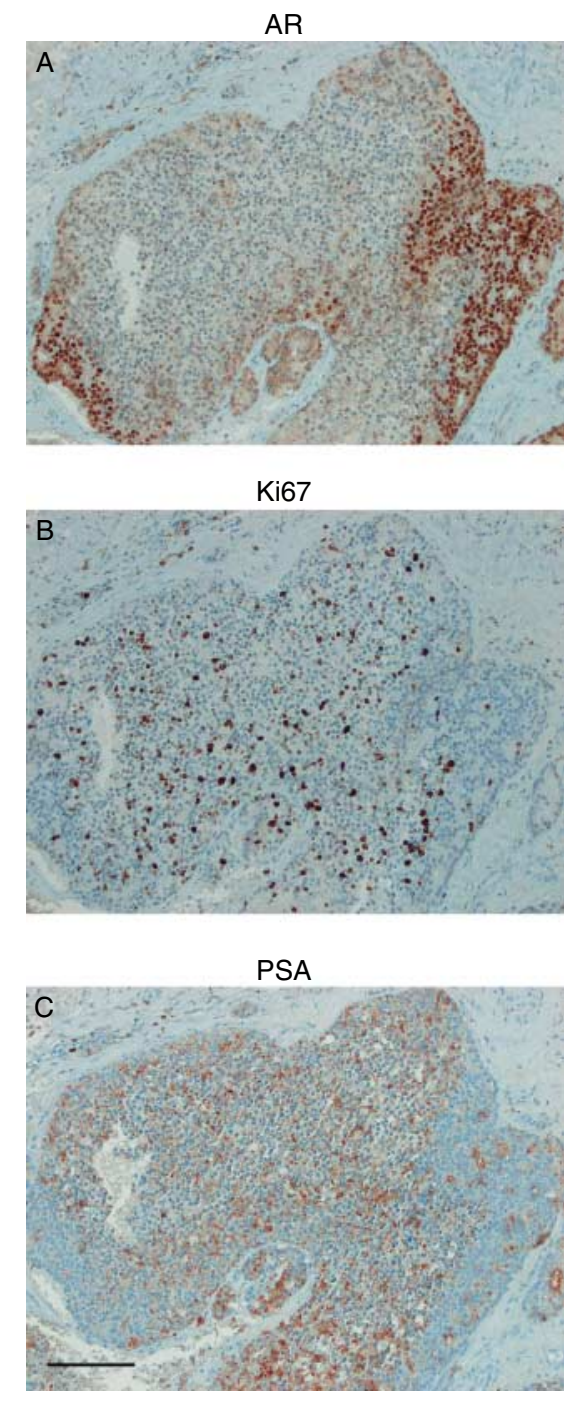

Figure 2 Staining for the androgen receptor (AR) (A), Ki67 (B) and prostate-specific antigen (PSA) (C) in consecutive sections from a single metastasis obtained from a patient with castration-resistant prostate cancer. In this case, high AR expressing areas show limited Ki67 and PSA staining. Bar indicates $100 \mu \mathrm{m}$. 

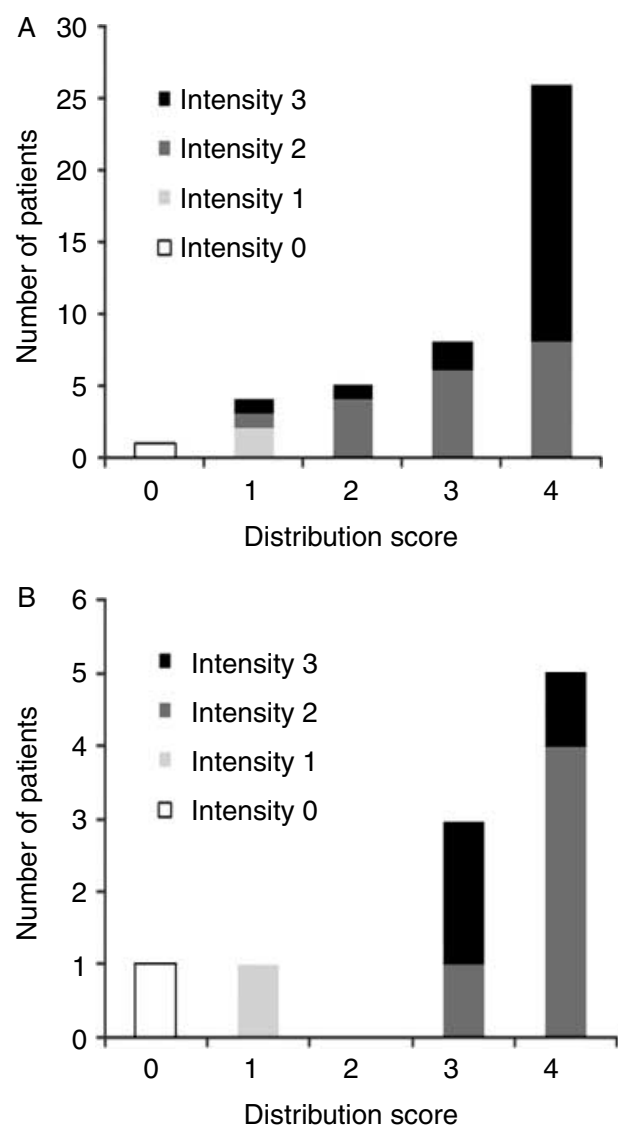

Figure 3 Histogram showing androgen receptor (AR) distribution and intensity in patients with castration-resistant $(A)$ and hormone-naive (B) prostate cancer bone metastases. Intensity score was as follows: 0 , no staining; 1 , weak; 2 , moderate; and 3 , intense staining. Distribution score was as follows: $0,0 \% ; 1$, $1-25 \%$; 2, 26-50\%; 3, 51-75\%; and 4, 76-100\%.

We then examined whether AR-high and AR-low CRPC patients differed in other aspects. This was, with some exceptions, apparently not the case (Table 3 ). The Ki67 labelling was significantly higher in AR-high patients than in AR-low patients. In AR-low patients, subsequent bisphosphonate treatment was more commonly used than in the AR-high group. We therefore analysed whether differences in treatment after metastasis surgery affected outcome in CRPC patients. Chemo- or radiation therapy did not influence outcome, but patients treated with bisphosphonates had an improved survival $(P=0.004$, data not shown) compared to the others. We thus excluded all cases given bisphosphonate after metastasis surgery from the Kaplan-Meier survival analysis. This still showed that AR-low patients had a better outcome than AR-high patients $(P=0.004$, data not shown $)$. ARs and bisphosphonate treatment were also independent predictors of outcome in a Cox survival analysis; risk ratios (RR) were $2.3(P=0.027$, confidence interval $(\mathrm{CI}): 1.1-4.8)$ and $0.3(P=0.013$, CI: $0.12-0.78)$ for AR-high and treatment with bisphosphonates respectively.

As suspected, high serum PSA (above median; $190 \mathrm{ng} / \mathrm{ml}$ ) at metastasis surgery was related to a poor outcome in CRPC patients (Fig. 4). Serum PSA was, however, not correlated to findings in metastases, not even to the PSA staining score, neither in the whole group nor in the subgroups of hormone-naive or CRPC metastases (results not shown). Importantly, serum PSA was not correlated to AR staining, and they both provided additional prognostic information according to Cox analysis; $\mathrm{RR}$ were 2.5 ( $P=0.018, \mathrm{CI}$ : $1.2-5.2$ ) and $3.1(P=0.0024, \mathrm{CI}$ : $1.5-6.3)$ for AR-high and high serum PSA at surgery respectively.

\section{Nuclear AR immunostaining is associated with cell proliferation and the presence of NE tumour cells, but not with tumour cell apoptosis or PSA expression}

As AR was related to a poor outcome in this type of advanced CRPC patients, we wanted to know whether the AR staining score was related to possible downstream effects of the AR such as cell proliferation (Ki67), apoptosis (activated caspase-3) and PSA, or to the presence of tumour cells with NE differentiation.

As indicated in Table 3, nuclear AR staining was not related to apoptosis (neither when caspase-3 staining nor when nuclear morphology was used to identify apoptotic cells) or PSA staining but significantly correlated to tumour cell proliferation in the whole group of bone metastases as well as in CRPC metastases $\left(R_{\mathrm{s}}=0.37, P=0.003, n=59\right.$ and $R_{\mathrm{S}}=0.38$, $P=0.012, n=43)$. Furthermore, neither tumour cell proliferation nor apoptosis was related to outcome in the CRPC patients (data not shown). Notably, the correlation coefficient between the AR score and Ki67 labelling was low, and it was not common to find AR-negative areas with numerous proliferating tumour cells (Fig. 2). It was also possible to find tumour areas with high AR and Ki67 but low PSA.

As prostate tumour cells with NE differentiation are known to be AR-negative in primary tumours, this was investigated also in metastases. NE tumour cells, expressing chromogranin A, were observed in 16 of the metastases, but in most cases, the fraction of NE tumour cells was very low. The AR staining score and percentage of NE cells present were not correlated, and although the AR staining was generally low in the few patients with a substantial number of NE cells, low AR staining was also seen in metastases areas lacking NE cell differentiation. 
Table 2 Characteristics of primary tumours and metastases in hormone-naive, short-term castrated and castration-resistant prostate cancer patients. Values are given as median (25th; 75th percentiles)

\begin{tabular}{lcccc}
\hline & AR score & Apoptosis index $^{\mathrm{a}}$ & Proliferation index $^{\mathrm{b}}$ & PSA score $^{\mathrm{a}}$ \\
\hline Primary tumours $n=16$ & $12(8,12)$ & $1.5(1.0,1.8)$ & $6.2(4.3,8.9)$ & $12(12,12)$ \\
Hormone-naive metastases $n=11$ & $8(5,9)^{\mathrm{c}, \mathrm{e}}$ & $2.1(0.70,3.3)$ & $17(11,25)^{\mathrm{e}, \mathrm{h}}$ & $9(4,12)^{\mathrm{c}, \mathrm{e}}$ \\
Short-term-treated metastases $n=7$ & $3(2,4)^{\mathrm{c}, \mathrm{f}}$ & $6.6(2.9,13)^{\mathrm{c}, \mathrm{f}}$ & $14(11,20)$ & $4(1,12)$ \\
Castration-resistant metastases $n=44^{\mathrm{d}}$ & $8(6,12)^{\mathrm{d}, \mathrm{g}}$ & $1.4(0.7,2.5)^{\mathrm{d}, \mathrm{g}}$ & $14(8.6,22)^{\mathrm{d}, \mathrm{e}}$ & $8(4,12)^{\mathrm{d}, \mathrm{e}}$ \\
\hline
\end{tabular}

${ }^{a}$ Androgen receptor (AR) and prostate-specific antigen (PSA) immunohistochemical staining scores were assessed according to details described in the Materials and methods section.

${ }^{\mathrm{b}}$ Apoptosis and proliferation indices were assessed as fractions of apoptotic (active caspase-3-immunostained) and proliferating (Ki67-immunostained) tumour epithelial cells, according to details described in the Materials and methods section.

${ }^{\mathrm{c}, \mathrm{d}} \mathrm{P}<0.05$ and 0.01 respectively in relation to ${ }^{\mathrm{e}}$ primary tumours, ${ }^{\mathrm{f}}$ hormone-naive metastases and ${ }^{\mathrm{g}}$ short-term-treated metastases, according to the Mann-Whitney $U$ test. ${ }^{\mathrm{h}} P=0.065$.

\section{Castration therapy has a biphasic effect on AR staining in bone metastases}

In the seven cases examined 2-7 days after surgical castration, median nuclear AR staining score was decreased, the PSA score and the Ki67 labelling index unaffected and the apoptosis index increased as compared to the hormone-naive tumours (Table 2). In the two cases where we had access to paired biopsies taken before and 3 days after surgical castration, nuclear AR staining was markedly and PSA staining slightly reduced (Fig. 5), whereas apoptosis was increased 3.9- and 4.2-fold and the Ki67 labelling index reduced to 0.7 and 0.77 of the pre-treatment values. In metastases from CRPC patients, AR staining was again as high as in hormone-naive cases and thus higher than in short-term castrated patients (Table 2).

\section{AR staining is lower in metastases than in untreated primary tumours}

In the 14 CRPC patients where we had access to both the primary hormone-naive tumours and the CRPC bone metastases, the median nuclear AR staining ( $7 \mathrm{vs}$ $12, n=14, P=0.057)$, apoptosis (1.2 vs $1.5, n=13$, $P=0.059)$ and PSA staining ( 8 vs $12, n=14$, $P=0.023$ ) appeared to be lower in the metastases than in primary tumours. The median Ki67 labelling index (16 vs 6.2, $n=13, P=0.011)$ and Gleason score (data not shown) were, however, higher in metastases than in the corresponding untreated primary tumours. Similar results were found when comparing all primary hormone-naive tumours with all hormone-naive bone metastases (Table 2).

\section{Low tissue PSA staining was associated with an unfavourable outcome in CRPC patients}

In the majority of metastases, most tumour epithelial cells expressed PSA, but it was also possible to find PSA-negative tumour cells in many metastases.
As PSA is known to be an androgen-regulated gene, it was not surprising to find AR and PSA staining in the same tumour areas, but AR-negative, PSA-positive tumour areas (Fig. 2) were also observed.

A PSA staining score of 8 and below (median and below, $n=25$ ) was associated with an unfavourable outcome in CRPC patients (Fig. 4), but did not add prognostic information when analysed together with nuclear AR immunostaining and serum PSA in Cox survival analysis (data not shown). As almost all metastases were poorly differentiated, it was not possible to find a relation between tumour cell differentiation and PSA.

\section{Discussion}

We note, for the first time, that high nuclear AR immunostaining in bone metastases (seen in about $60 \%$ of CRPC cases) is associated with a particularly poor prognosis after metastasis surgery. This is in contrast to others who did not obtain any prognostic information from AR staining (Cheville et al. 2002). Our finding is, however, in line with observations in primary prostate tumours (Henshall et al. 2001, Li et al. 2004) and in lymph node metastases (Sweat et al. 1999), and the fact that $\mathrm{AR}$ transcriptional activity is reactivated in most CRPC patients (Chen et al. 2008). As high nuclear AR staining predicts an unfavourable outcome in CRPC patients, this may suggest that this subset of patients could benefit from novel drugs that effectively block AR signalling (Tran et al. 2009). However, as patients with constitutive active ARs (Guo et al. 2009, Hu et al. 2009) are probably detected by the AR antibodies used in this study, it is considered that not all men with high staining may be responders to this type of treatment. Accordingly, treatment with MVD3100 resulted in a marked response in $40 \%$ of CRPC patients (Tran et al. 2009). Whether potential responders can be identified by AR expression levels in metastases therefore 
Table 3 Characteristics of castration-resistant prostate cancer patients and their bone metastases stratified according to nuclear androgen receptor (AR) staining. Continuous values are given as median (25th; 75 th percentiles)

\begin{tabular}{|c|c|c|c|}
\hline & AR-high $^{a}$ & AR-low ${ }^{a}$ & $P$ value \\
\hline Age at surgery & $69(66,80)$ & $73(70,76)$ & NS \\
\hline $\begin{array}{l}\text { Serum PSA at } \\
\text { surgery }(\mathrm{ng} / \mathrm{ml})\end{array}$ & $236(66,891)$ & $145(96,573)$ & NS \\
\hline PSA score & $8(6,12)$ & $9(6,12)$ & NS \\
\hline Ki67 index (\%) & $15(11,24)$ & $10(5.1,16)$ & 0.023 \\
\hline Apoptosis index (\%) & $1.5(1.0,2.7)$ & $1.2(0.65,2.1)$ & NS \\
\hline Chromogranin A (\%) & $0(0,1.4)$ & $0(0,0)$ & NS \\
\hline \multicolumn{4}{|l|}{ Pre surgery $(n)$} \\
\hline Castration $^{\mathrm{b}}$ & 18 & 14 & NS \\
\hline $\begin{array}{l}\text { Castration plus } \\
\text { anti-androgen }\end{array}$ & 10 & 2 & NS \\
\hline Radiation $^{d}$ & 5 & 2 & NS \\
\hline Chemotherapy & 5 & 1 & NS \\
\hline Bisphosphonates & 2 & 1 & NS \\
\hline \multicolumn{4}{|l|}{ Post surgery $(n)$} \\
\hline Chemotherapy & 6 & 4 & NS \\
\hline Radiation $^{\mathrm{e}}$ & 14 & 11 & NS \\
\hline Bisphosphonate $^{f}$ & 3 & 6 & 0.034 \\
\hline
\end{tabular}

NS, non-significant according to Mann-Whitney $U$ test or $\chi^{2}$-test.

${ }^{a}$ AR-high was defined as a nuclear AR immunostaining of median or above and AR-low as a score below median.

${ }^{b}$ Includes surgical ablation, LHRH/GnRH agonist therapy and previous therapy with anti-androgens (bicalutamide and flutamide), but specifically no anti-androgens at the time for orthopaedic surgery.

${ }^{\circ}$ Castration therapy as stated above and anti-androgen therapy (bicalutamide) at the time of orthopaedic surgery.

${ }^{\mathrm{d}}$ Radiation towards operation site.

${ }^{\mathrm{e}}$ Radiation towards any site after surgery.

fBisphosphonate treatment was started 1-18 months after metastasis surgery.

remains to be explored. Unfortunately, our results indicate that AR levels in metastases cannot be predicted by measuring serum PSA.

Androgens are known to affect cell proliferation and cell death in PC cells (Gao et al. 2001, Denmeade \& Isaacs 2002), but in this study, neither tumour cell proliferation nor apoptosis in the bone metastases was related to clinical outcome. Similarly, tumour cell Ki67 labelling in bone metastases was unrelated to prognosis in a previous study (Cheville et al. 2002). Factors apart from AR-regulated metastasis growth thus probably explain why some patients at this late and particularly advanced stage of the disease die earlier than others. Such factors could be AR-stimulated secretion of inflammation mediators, such as interleukin- 6 or tumour necrosis factor- $\alpha$, causing cancer cachexia (Pfitzenmaier et al. 2003, Kuroda et al. 2005). Notably, intense nuclear AR staining does not necessarily mean that all possible downstream targets are activated.
For example, some tumour areas with intense nuclear AR staining did not express PSA, and the cells did not always proliferate. Whether some of these findings can be explained by the presence of constitutively active AR variants that, in contrast to wild-type ARs, may or may not stimulate PSA synthesis (Guo et al. 2009, Hu et al. 2009) remains to be explored. AR staining was, however, positively correlated to cell proliferation, but the correlation coefficient was not high, suggesting that metastasis growth is stimulated by AR activation (Gao et al. 2001) and by other factors. Notably, however, high nuclear AR staining does not necessarily mean that AR signalling is active, and it is also possible that the antibody does not recognize all forms of active ARs.

A substantial number of metastatic cells expressed low AR levels in a minor or major subpopulation of the tumour cells, as already described (Hobisch et al. 1995, Shah et al. 2004). CRPC patients with low AR staining score had a better prognosis than the others. For reasons that are unknown, these men who were in our set of patients were more often treated with bisphosphonates after metastasis surgery, and as this treatment may prolong survival (Dearnaley et al. 2009), it could be argued that differences in survival between AR-low and AR-high patients could be explained by differences in postoperative treatment. This is, however, not the case, as low AR staining was also a marker of more favourable outcome when bisphosphonate-treated patients were excluded. When we examined the patient records, we noted that postoperative bisphosphonate treatment was not given until months after metastasis surgery, i.e. only in patients who already appeared to have the best outcome. NE and stem cell-like tumour cells lack AR expression (Maitland \& Collins 2008), and in our study, metastases with a particularly high fraction of NE cells had a low AR staining score. However, low AR staining could not be explained only by the presence of NE cells or stem cell-like tumour cells, as such cell types are, as already reported (Roudier et al. 2003, Eaton et al. 2010), generally rare in bone metastases. As also patients with low AR staining eventually die from CRPC, it is likely that factors apart from ARs drive metastases growth and secretion of cachexia-causing substances in such tumours, and that these patients need therapies other than AR blockade.

The standard procedure to follow effects of treatment in bone metastases is by monitoring changes in serum PSA. Not surprisingly, a high pre-operative serum PSA level was related to an unfavourable outcome. More surprising are the observations in this and previous studies that PSA staining in metastases is 

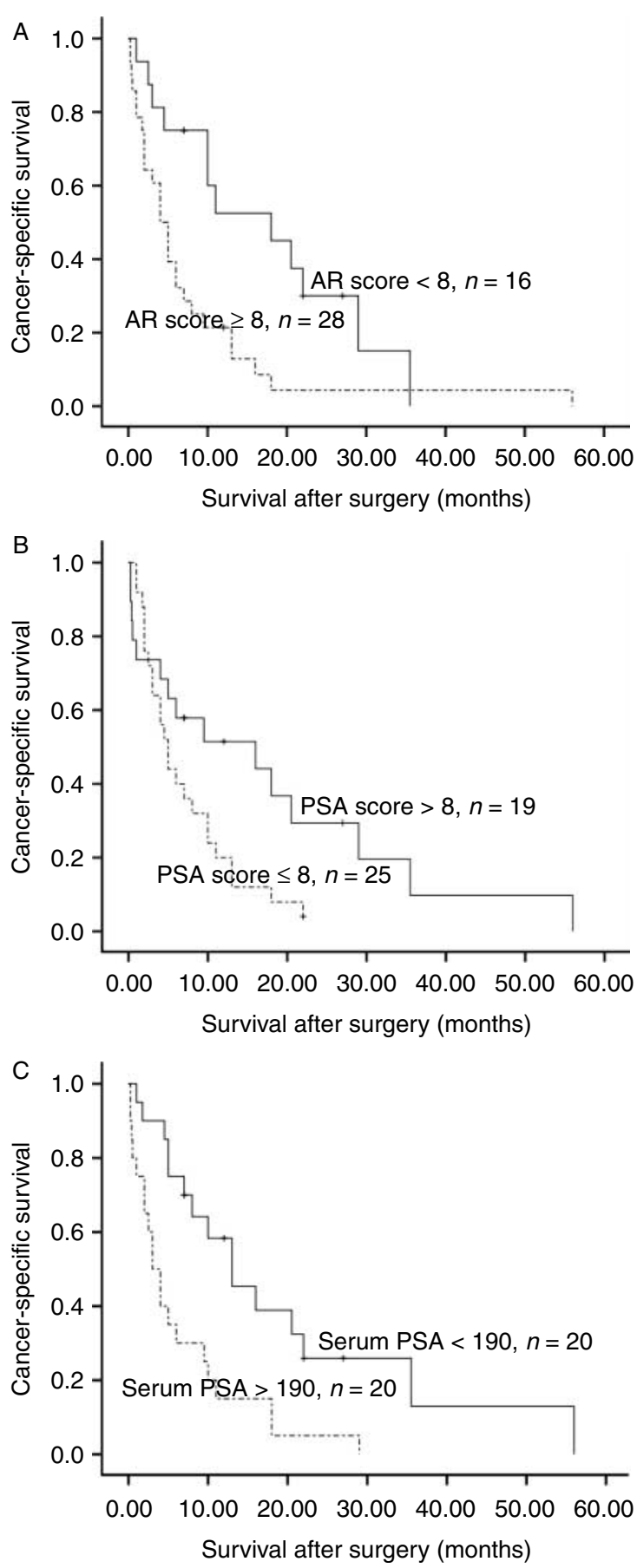

Figure 4 Kaplan-Meier analysis of androgen receptor (AR) staining, prostate-specific antigen (PSA) staining and serum $P S A$ in relation to cancer-specific survival after surgery for spinal cord compression or pathological fractures in patients with castration-resistant prostate cancer. An AR staining score of 8 or above (A, $P=0.008)$, PSA staining score of 8 or below $(B, P=0.046)$ and serum PSA above median, $190 \mathrm{ng} / \mathrm{ml}$ (C, $P=0.002)$, were all related to a poor outcome. generally lower than in primary tumours (Hobisch et al. 1995, Cheville et al. 2002, Roudier et al. 2003, Shah et al. 2004), and that it is not correlated to serum PSA (Roudier et al. 2003, Shah et al. 2004), AR expression (Hobisch et al. 1995, Cheville et al. 2002) or tumour cell proliferation. Similarly, tumour cell PSA, ARs and proliferation were not correlated in primary tumours, and they were all unrelated to serum PSA (Ohlson et al. 2005, 2006, 2007). To follow changes in serum PSA, although PSA levels are related to prognosis, is apparently not an ideal way to monitor changes within tumour cells. Interestingly, a high PSA staining score in metastases was associated with favourable outcome as previously observed both in metastases (Cheville et al. 2002, Roudier et al. 2003, Shah et al. 2004) and in primary tumours (Stege et al. 2000, Ohlson et al. 2005), possibly as it indicates some degree of tumour cell differentiation.

Although castration has been the standard treatment against PC bone metastases for the last 60 years, the short-term morphological effects of this treatment have not been examined in patients. In bone metastases, surgical castration induced an initial drop in nuclear AR levels (probably induced by a temporary decrease in androgen levels in metastases, Wikstrom et al. (2007) and Montgomery et al. (2008)) and an increase in tumour cell apoptosis, but only a moderate decrease in cell proliferation. In our previous studies of primary tumours, nuclear AR staining was decreased and proliferation markedly reduced (to 0.1 of pre-treatment values), while apoptosis was only moderately (two- to threefold) increased 3-7 days after treatment (Ohlson et al. 2005, 2006, 2007). It thus seems, at least in the limited number of patents examined, that castration causes cell death in metastases of at least the same magnitude as in primary tumours, but the inhibition of cell proliferation is probably less efficient. Different responses in primary and metastatic tumour cells may explain why relapse occurs more commonly in metastases than in primary tumours in patients (Ohlson et al. 2006) and in animal models (Wikstrom et al. 2005). The most likely cause to such differences is the microenvironment. Castration treatment of the normal prostate and primary prostate tumours works in part by affecting AR-positive cells in the stroma and vasculature (Johansson et al. 2007, Ohlson et al. 2007), and understanding the hormonal regulation of the metastasis stroma is therefore important. Many cell types in the bone/bone marrow express ARs (Abu et al. 1997, Mantalaris et al. 2001), but we found few AR-positive cells in the metastasis microenvironment. The reason to this is unknown. In aggressive primary tumours, the development of an AR-negative stroma is associated 

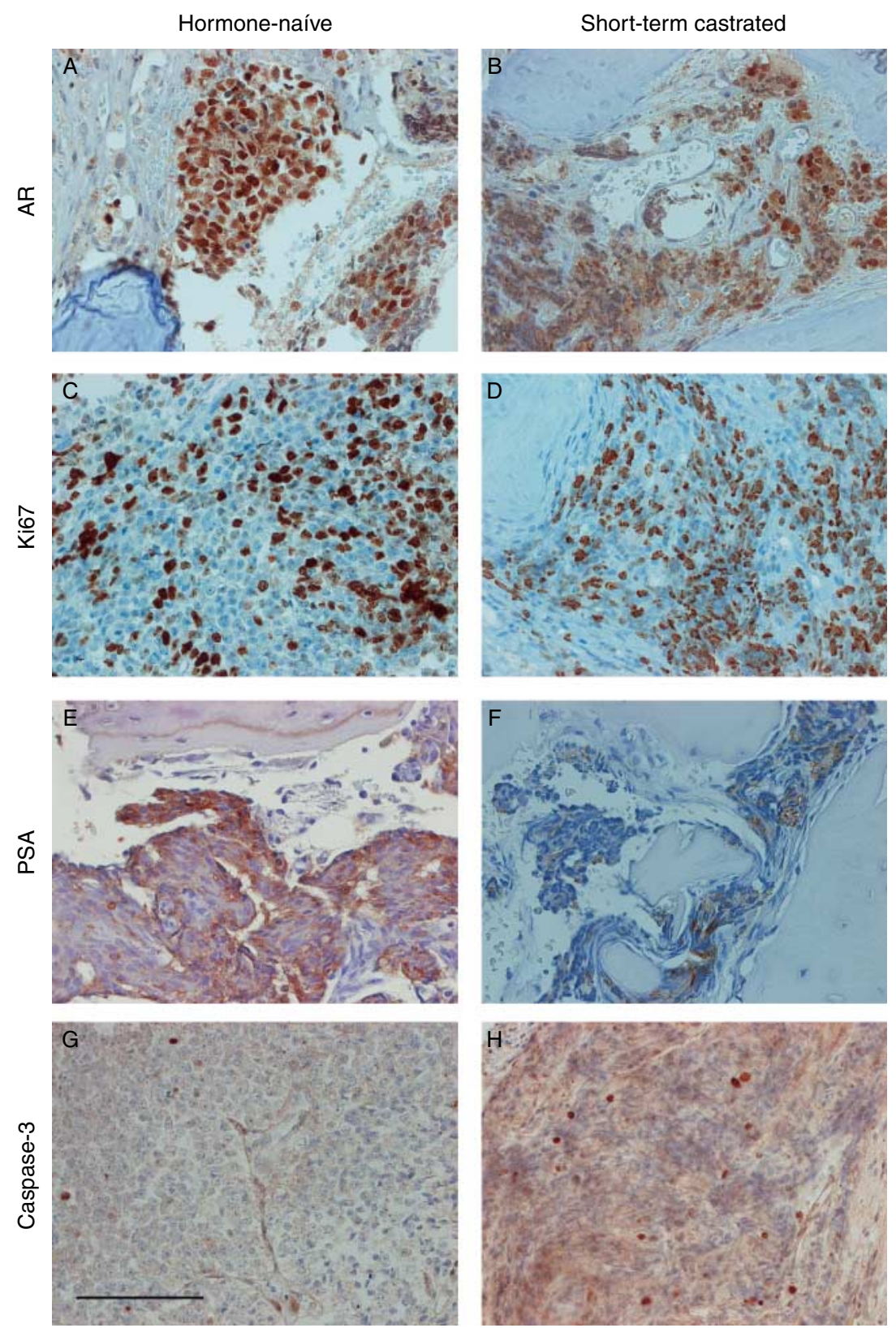

Figure 5 Sections from a bone metastasis taken before $(A, C, E$ and $G)$ and 3 days after castration therapy $(B, D, F$ and $H)$ in one patient. Short-term castration therapy reduced androgen receptor (AR) nuclear staining and to some extent also PSA staining, increased caspase-3 (apoptosis) staining, whereas Ki67 staining was largely unaffected. Bar indicates $100 \mu \mathrm{m}$.

with a poor response to androgen ablation therapy (Wikstrom et al. 2009), and similar mechanism may be operating in metastases. Studies of how androgen ablation therapy really works in bone metastases are needed in order to find novel ways to enhance the efficacy of this treatment.

In conclusion, our study suggests that nuclear AR immunostaining in CRPC bone metastases is, in contrast to tumour cell proliferation and apoptosis, associated with a poor clinical outcome. As nuclear AR levels were not associated with intracellular PSA levels (a welldefined androgen-regulated gene), it is possible that active AR signalling in metastases in CRPC cases induces responses different from those in normal prostate cells. These putative downstream effects of the ARs, responsible for poor outcome, need to be defined. 


\section{Supplementary data}

This is linked to the online version of the paper at http://dx. doi.org/10.1677/ERC-10-0059.

\section{Declaration of interest}

The authors declare that there is no conflict of interest that could be perceived as prejudicing the impartiality of the research reported.

\section{Funding}

This work was supported by grants from the Swedish Cancer Society, Swedish Research Council, The county of Västerbotten, Lions Cancer Research foundation and Umeå University.

\section{Acknowledgements}

Skilful technical assistance was provided by Ms Pernilla Andersson, Elisabeth Dahlberg, Birgitta Ekblom, Inger Lindström and Åsa Skytt.

\section{References}

Abu EO, Horner A, Kusec V, Triffitt JT \& Compston JE 1997 The localization of androgen receptors in human bone. Journal of Clinical Endocrinology and Metabolism 82 3493-3497. (doi:10.1210/jc.82.10.3493)

Asmann YW, Kosari F, Wang K, Cheville JC \& Vasmatzis G 2002 Identification of differentially expressed genes in normal and malignant prostate by electronic profiling of expressed sequence tags. Cancer Research 62 3308-3314.

Bonkhoff H \& Berges R 2010 From pathogenesis to prevention of castration resistant prostate cancer. Prostate 70 100-112. (doi:10.1002/pros.21042)

Chen CD, Welsbie DS, Tran C, Baek SH, Chen R, Vessella R, Rosenfeld MG \& Sawyers CL 2004 Molecular determinants of resistance to antiandrogen therapy. Nature Medicine 10 33-39. (doi:10.1038/nm972)

Chen Y, Sawyers CL \& Scher HI 2008 Targeting the androgen receptor pathway in prostate cancer. Current Opinion in Pharmacology 8 440-448. (doi:10.1016/j. coph.2008.07.005)

Cheville JC, Tindall D, Boelter C, Jenkins R, Lohse CM, Pankratz VS, Sebo TJ, Davis B \& Blute ML 2002 Metastatic prostate carcinoma to bone: clinical and pathologic features associated with cancer-specific survival. Cancer 95 1028-1036. (doi:10.1002/cncr.10788)

Dearnaley DP, Mason MD, Parmar MK, Sanders K \& Sydes MR 2009 Adjuvant therapy with oral sodium clodronate in locally advanced and metastatic prostate cancer: long-term overall survival results from the MRC PR04 and PR05 randomised controlled trials. Lancet Oncology 10 872-876. (doi:10.1016/S1470-2045(09)70201-3)
Denmeade SR \& Isaacs JT 2002 A history of prostate cancer treatment. Nature Reviews. Cancer 2 389-396. (doi:10. 1038/nrc801)

Eaton CL, Colombel M, van der Pluijm G, Cecchini M, Wetterwald A, Lippitt J, Rehman I, Hamdy F \& Thalman G 2010 Evaluation of the frequency of putative prostate cancer stem cells in primary and metastatic prostate cancer. Prostate 70 875-882. (doi:10.1002/pros.21121)

Gao J, Arnold JT \& Isaacs JT 2001 Conversion from a paracrine to an autocrine mechanism of androgenstimulated growth during malignant transformation of prostatic epithelial cells. Cancer Research 61 5038-5044.

Guo Z, Yang X, Sun F, Jiang R, Linn DE, Chen H, Kong X, Melamed J, Tepper CG, Kung HJ et al. 2009 A novel androgen receptor splice variant is up-regulated during prostate cancer progression and promotes androgen depletion-resistant growth. Cancer Research 69 2305-2313. (doi:10.1158/0008-5472.CAN-08-3795)

Henshall SM, Quinn DI, Lee CS, Head DR, Golovsky D, Brenner PC, Delprado W, Stricker PD, Grygiel JJ \& Sutherland RL 2001 Altered expression of androgen receptor in the malignant epithelium and adjacent stroma is associated with early relapse in prostate cancer. Cancer Research 61 423-427.

Hobisch A, Culig Z, Radmayr C, Bartsch G, Klocker H \& Hittmair A 1995 Distant metastases from prostatic carcinoma express androgen receptor protein. Cancer Research 55 3068-3072.

Holzbeierlein J, Lal P, LaTulippe E, Smith A, Satagopan J, Zhang L, Ryan C, Smith S, Scher H, Scardino P et al. 2004 Gene expression analysis of human prostate carcinoma during hormonal therapy identifies androgenresponsive genes and mechanisms of therapy resistance. American Journal of Pathology 164 217-227.

Hu R, Dunn TA, Wei S, Isharwal S, Veltri RW, Humphreys E, Han M, Partin AW, Vessella RL, Isaacs WB et al. 2009 Ligand-independent androgen receptor variants derived from splicing of cryptic exons signify hormone-refractory prostate cancer. Cancer Research 69 16-22. (doi:10.1158/ 0008-5472.CAN-08-2764)

Johansson A, Jones J, Pietras K, Kilter S, Skytt A, Rudolfsson SH \& Bergh A 2007 A stroma targeted therapy enhances castration effects in a transplantable rat prostate cancer model. Prostate 67 1664-1676. (doi:10.1002/pros.20657)

Kuroda K, Horiguchi Y, Nakashima J, Kikuchi E, Kanao K, Miyajima A, Ohigashi T, Umezawa K \& Murai M 2005 Prevention of cancer cachexia by a novel nuclear factor \{kappa\}B inhibitor in prostate cancer. Clinical Cancer Research 11 5590-5594. (doi:10.1158/1078-0432.CCR04-2561)

Li R, Wheeler T, Dai H, Frolov A, Thompson T \& Ayala G 2004 High level of androgen receptor is associated with aggressive clinicopathologic features and decreased biochemical recurrence-free survival in prostate: cancer patients treated with radical prostatectomy. American Journal of Surgical Pathology 28 928-934. (doi:10.1097/ 00000478-200407000-00013) 
Maitland NJ \& Collins AT 2008 Prostate cancer stem cells: a new target for therapy. Journal of Clinical Oncology 26 2862-2870. (doi:10.1200/JCO.2007.15.1472)

Mantalaris A, Panoskaltsis N, Sakai Y, Bourne P, Chang C, Messing EM \& Wu JH 2001 Localization of androgen receptor expression in human bone marrow. Journal of Pathology 193 361-366. (doi:10.1002/10969896(0000)9999:9999<::AID-PATH803> 3.0.CO;2-W)

Montgomery RB, Mostaghel EA, Vessella R, Hess DL, Kalhorn TF, Higano CS, True LD \& Nelson PS 2008 Maintenance of intratumoral androgens in metastatic prostate cancer: a mechanism for castration-resistant tumor growth. Cancer Research 68 4447-4454. (doi:10. 1158/0008-5472.CAN-08-0249)

Morrissey C \& Vessella RL 2007 The role of tumor microenvironment in prostate cancer bone metastasis. Journal of Cellular Biochemistry 101 873-886. (doi:10. 1002/jcb.21214)

Ohlson N, Wikstrom P, Stattin P \& Bergh A 2005 Cell proliferation and apoptosis in prostate tumors and adjacent non-malignant prostate tissue in patients at different time-points after castration treatment. Prostate 62 307-315. (doi:10.1002/pros.20139)

Ohlson N, Bergh A, Nygren K, Stattin P \& Wikstrom P 2006 The magnitude of early castration-induced primary tumour regression in prostate cancer does not predict clinical outcome. European Urology 49 675-684. (doi:10. 1016/j.eururo.2005.10.024)

Ohlson N, Bergh A, Stattin P \& Wikstrom P 2007 Castrationinduced epithelial cell death in human prostate tissue is related to locally reduced IGF-1 levels. Prostate 67 32-40. (doi:10.1002/pros.20480)

Pfitzenmaier J, Vessella R, Higano CS, Noteboom JL, Wallace D Jr \& Corey E 2003 Elevation of cytokine levels in cachectic patients with prostate carcinoma. Cancer $\mathbf{9 7}$ 1211-1216. (doi:10.1002/cncr.11178)

Roudier MP, True LD, Higano CS, Vesselle H, Ellis W, Lange P \& Vessella RL 2003 Phenotypic heterogeneity of end-stage prostate carcinoma metastatic to bone. Human Pathology 34 646-653. (doi:10.1016/S00468177(03)00190-4)
Shah RB, Mehra R, Chinnaiyan AM, Shen R, Ghosh D, Zhou M, Macvicar GR, Varambally S, Harwood J, Bismar TA et al. 2004 Androgen-independent prostate cancer is a heterogeneous group of diseases: lessons from a rapid autopsy program. Cancer Research 64 9209-9216. (doi:10.1158/0008-5472.CAN-04-2442)

Stanbrough M, Bubley GJ, Ross K, Golub TR, Rubin MA, Penning TM, Febbo PG \& Balk SP 2006 Increased expression of genes converting adrenal androgens to testosterone in androgen-independent prostate cancer. Cancer Research 66 2815-2825. (doi:10.1158/00085472.CAN-05-4000)

Stege R, Grande M, Carlstrom K, Tribukait B \& Pousette A 2000 Prognostic significance of tissue prostate-specific antigen in endocrine-treated prostate carcinomas. Clinical Cancer Research 6 160-165.

Sweat SD, Pacelli A, Bergstralh EJ, Slezak JM, Cheng L \& Bostwick DG 1999 Androgen receptor expression in prostate cancer lymph node metastases is predictive of outcome after surgery. Journal of Urology 161 1233-1237. (doi:10.1016/S0022-5347(01)61645-8)

Tran C, Ouk S, Clegg NJ, Chen Y, Watson PA, Arora V, Wongvipat J, Smith-Jones PM, Yoo D, Kwon A et al. 2009 Development of a second-generation antiandrogen for treatment of advanced prostate cancer. Science $\mathbf{3 2 4}$ 787-790. (doi:10.1126/science.1168175)

Wikstrom P, Lindahl C \& Bergh A 2005 Characterization of the autochthonous transgenic adenocarcinoma of the mouse prostate (TRAMP) as a model to study effects of castration therapy. Prostate 62 148-164. (doi:10.1002/ pros.20123)

Wikstrom P, Ohlson N, Stattin P \& Bergh A 2007 Nuclear androgen receptors recur in the epithelial and stromal compartments of malignant and non-malignant human prostate tissue several months after castration therapy. Prostate 67 1277-1284. (doi:10.1002/pros. 20569)

Wikstrom P, Marusic J, Stattin P \& Bergh A 2009 Low stroma androgen receptor level in normal and tumor prostate tissue is related to poor outcome in prostate cancer patients. Prostate 69 799-809. (doi:10.1002/pros.20927) 